\title{
Crocetin improves ischaemic stroke in vitro and vivo
}

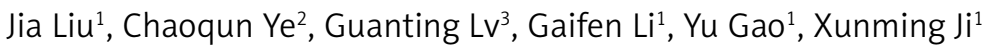

${ }^{1}$ China-America Institute of Neuroscience, Xuanwu Hospital, Capital Medical University, Beijing, China

2Department of Rehabilitation Medicine, Air Force Medical Center, PLA, Beijing, China ${ }^{3}$ Department of Blood Transfusion, Tangdu Hospital, Air Force Medical University, Xi'an, China

Submitted: 2 March 2021

Accepted: 20 March 2021

Arch Med Sci

DOI: https://doi.org/10.5114/aoms/133886

Copyright (c) 2021 Termedia \& Banach

\section{Abstract}

Introduction: This study aimed to investigate the preventive and therapeutic effects of crocetin (Cro) on ischaemic stroke in cell and animal models.

Material and methods: A cell model of oxygen and glucose deprivation (OGD) and a rat model of middle cerebral artery occlusion were established to simulate ischaemic stroke. The infarct volume was measured by TTC assay, and the apoptotic cell number was counted by TUNEL. Relative protein and gene expression levels in the rats were measured by immunohistochemical and RT-qPCR assays. The apoptosis rate and relative protein and gene expression levels were determined by flow cytometry, WB and RT-qPCR assay, respectively.

Results: Compared with those in the normal control (NC) group, the brain tissue injury and apoptotic cell number significantly increased $(p<0.001)$ and the miR-145-5p gene expression significantly decreased in the cell and animal experiments. In the animal experiment, the infarct volume, apoptotic cell number and pathological status improved in the Cro-treated groups. In the cell experiment, the apoptosis rates significantly depressed in the Cro-treated groups $(p<0.05)$. However, the cell apoptosis rate significantly increased after miR-145-5p inhibitor transfection $(p<0.001)$. The protein and gene expression levels of Toll-like receptor 4 , myeloid differentiation factor 88 and nuclear factor (NF)- $\kappa B$ (p65) significantly decreased $(p<0.05)$. In addition, $\mathrm{p}-\mathrm{NF}-\kappa \mathrm{B}(\mathrm{p} 65)$ nuclear volume significantly decreased $(p<0.05)$. Conclusions: Crocetin improved ischaemic stroke by regulating the miR145-5p/TLR4 axis in cell and animal experiments.

Key words: crocetin, ischaemic stroke, miR-145-5p, TLR4, cell, animal.

\section{Introduction}

Cerebral ischaemic stroke (CIS) is the leading cause of death and disability among adults in China. Specifically, $70-80 \%$ of CIS patients may lose the ability to live independently because of this disease [1, 2]. Normal neurons may lose function within seconds and be subject to structural changes within 2 min after a stroke. Moreover, the imbalance of ions and water inside and outside the neuron membrane is caused by energy supply disorders and transmembrane ion channel abnormalities, causing neuronal apoptosis and necrosis and generating corresponding symptoms and signs [3].

Crocetin (Cro) is a major chemical component of the stigma of Crocus sativus belonging to the family Iridaceae. Pharmacological studies proved 
that Cro exerts anti-atherosclerotic [4] and anti-hypertensive effects and alleviates cerebral ischaemia reperfusion injury $[5,6]$. Nevertheless, the mechanism of action of Cro in CIS remains unclear.

Toll-like receptor 4 (TLR4) is an innate immune receptor which activates the downstream myeloid differentiation factor 88 (MyD88)-dependent/nonMyD88-dependent signalling pathway by combining with the corresponding damage-associated molecular patterns (DAMPs) and then activates nuclear factor- $\kappa \mathrm{B}(\mathrm{NF}-\kappa \mathrm{B})$ to regulate the immune response and initiate the maintenance of secondary injury [7].

Micro-RNAs (miRNAs) are small non-coding RNAs which play a vital role in many biological processes, such as cell migration, invasion, proliferation and apoptosis. MiRNAs are closely related to the development and progression of CIS. Therefore, miRNAs can be used as a potential target for the diagnosis and treatment of CIS [8]. Zhang et al. [9] reported that the expression of miR-146a$5 p$ in CIS is unusually low. However, the therapeutic mechanism of miRNAs in CIS remains unclear.

In this study, the effects of different doses of Cro on animal and cell models of CIS were observed, and the changes in relevant miRNAs were detected. Subsequently, the role of miR-145-5p in improving CIS by Cro was tested in a cell experiment, and the correlation between miR-145-5p and TLR4 was verified in a dual-luciferase target experiment to analyse further the mechanism of action of Cro.

\section{Material and methods}

\section{Experimental materials}

Cro (Sigma, USA) was prepared in a $0.5 \%$ suspension with $0.5 \%$ carboxyl methyl cellulose sodium (CMC-Na) before use. The other materials used were as follows: SABC immunohistochemical kit (Wuhan BOSTER Biological Technology Co., Ltd., Wuhan, China) for TLR4, MyD88 and NF-кB (p65); rabbit anti-rat polyclonal primary antibody

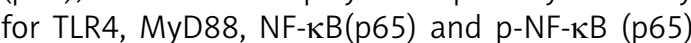
(Abcam, Cambridge, UK); flow apoptosis kit (Nanjing KeyGen BioTECH Co., Ltd., Nanjing, China); miR-145-5p inhibitor (Wuhan BOSTER Biological Technology Co., Ltd., Wuhan, China); Lipofectamine 2000 transfection reagents (Shanghai Kemin Bio-Tech Co., Ltd., Shanghai, China); double luciferase reporter gene detection kit (Beijing YPH Bio-Tech Co., Ltd., Beijing, China) and BCA kit (Shanghai Yise Medical Technology Co., Ltd., Shanghai, China).

\section{Cell culture and treatment}

A cell model of oxygen and glucose deprivation (OGD) was established using human U87 glioma cells to simulate cerebral ischaemia in vitro
[10]. The cells were cultured with sugar-free culture-medium in the absence of oxygen $\left(95 \% \mathrm{~N}_{2}\right.$ $+5 \%\left(\mathrm{CO}_{2}\right)$. Cro of different concentrations was used for intervention, and the cells were divided into Cro-L (5 mg/l), Cro-M (10 mg/l) and Cro-H $(50 \mathrm{mg} / \mathrm{l})$ groups based on the difference in Cro concentration. The normal control (NC) group was cultured with normal aerobic and sugar-containing medium, and the model group was not subject to any treatment. Cells in all groups received the corresponding treatment for $48 \mathrm{~h}$, after which subsequent experiments were carried out.

\section{Cell transfection}

When the cell growth reached $70 \%$ fusion, the serum-free medium was replaced. Lipofectamine 2000 was used to transfect miR-145-5p inhibitor into the cells. Fresh medium was used $6 \mathrm{~h}$ after the transfection for further culture, and the next experiment was carried out.

\section{Animal experiment}

SPF rats weighing 200-250 g were selected to establish a middle cerebral artery occlusion (MCAO) model. The occlusion with a length of $25 \mathrm{~mm}$ and a diameter of $0.2 \pm 0.01$ was placed in $1: 2500$ units of heparin saline for subsequent use. The common carotid artery, external carotid artery (ECA) and internal carotid artery (ICA) on one side of the rats were identified and dissociated; the ECA was ligated and cut off. The occlusion was inserted into the ECA and reached the middle cerebral artery via the ICA. The insertion was stopped when encountering resistance. The occlusion was inserted to a depth of about $10 \mathrm{~mm}$. Thus, the rat model of MCAO was completed. After 60 min of ischaemia, the occlusion was withdrawn from the ICA to achieve blood recirculation, thus completing blood reperfusion. The rats were randomly divided into an NC group, a model group, a Cro-L group (5 mg/kg Cro), a Cro-M (10 mg/kg Cro) group and a Cro-H (50 mg/kg Cro) group. The $\mathrm{NC}$ and model groups with nine rats each were intragastrically administered with an equal volume of distilled water. The rats in the five groups received gavage treatment for 10 days. Then, the rats in the model, Cro-L, Cro-M and Cro-H groups were treated by MCAO for $60 \mathrm{~min}$, after which reperfusion was performed for $24 \mathrm{~h}$. This study was approved by the ethics committee of Xuanwu Hospital (approval No. 2019-03-049). The entire experimental process followed the Regulations on the Administration of Laboratory Animals formulated by the Science and Technology Commission of the People's Republic of China, and the Guiding Opinions on the Good Treatment of Laboratory Animals issued by the Ministry of Science and Technology of the People's Republic of China. 
Measurement of cerebral infarction volume of rats in each group by TTC staining

Rats were anaesthetised and killed $24 \mathrm{~h}$ after reperfusion. Their brains were quickly extracted and then were placed in $\mathrm{a}-20^{\circ} \mathrm{C}$ refrigerator for $15 \mathrm{~min}$. The cerebellum and brainstem were removed. Five consecutive $2 \mathrm{~mm}$-thick tissues were sectioned along the coronal plane of the brain. The sections were placed in $2 \%$ TTC phosphate-balanced solution and in $37^{\circ} \mathrm{C}$ water bath for staining in a dark place for $20 \mathrm{~min}$. The brain tissues were gently turned once every $5 \mathrm{~min}$. After the staining, the tissues were placed in $4 \%$ paraformaldehyde solution for fixation. After $24 \mathrm{~h}$, each section of brain tissue was photographed. The infarction area was measured using Image-pro plus 6.0.

\section{HE staining}

The brain tissue was fixed in $4 \%$ paraformaldehyde for 2-3 days, dehydrated by gradient alcohol and then embedded with transparent xylene and paraffin. Tissue samples with a thickness of $4 \mu \mathrm{m}$ were sectioned along the optic chiasma plane and the coronal plane for HE staining. Five non-overlapping fields of vision in the ischaemic lateral cortical area were randomly taken from the sections under the microscope $(10 \times 20)$ to observe the damaged cells. Such cells had cavitation-like denaturation, eosinophilic denaturation, nuclear shrinkage, nuclear dissolution and other changes.

\section{TUNEL staining}

The chest was opened to expose the heart. The auricula dextra was dissected. An indwelling needle was inserted at the apex cordis and connected to a syringe to inject $300 \mathrm{ml}$ of normal saline at a constant speed until the fluid from the right auricle was bloodless, the limbs and lungs turned white and the liver became yellow. Then, $300 \mathrm{ml}$ of paraformaldehyde was injected until the neck and limbs of the rats were stiffened and the internal organs turned white. At this moment, the rats were quickly decapitated to extract the brain tissue, which was fixed in paraformaldehyde for $24 \mathrm{~h}$. The brain tissue was dehydrated and embedded with paraffin and dissected into $5 \mu \mathrm{m}$-thick sections. The paraffin sections were dewaxed to water. Citrate buffer solution was added for antigen retrieval. The sections were sealed with a sealant containing sheep serum for $2 \mathrm{~h}$, the primary antibody TUNEL (diluted by $1: 600$ ) was added, incubated overnight, the secondary antibody (diluted by $1: 1000$ ) was added and then incubated for $2 \mathrm{~h}$. DAPI staining solution was added for $8 \mathrm{~min}$. An anti-quenching agent was added. Observation under an optical microscope was performed. Five cerebral ischaemic lateral brain tissues were tak- en in a 200-fold field of view and photographed to calculate the number of apoptotic cells in each group.

\section{Immunohistochemical (IHC) staining}

The tissue specimens were embedded and sectioned, dewaxed with xylene and then hydrated with gradient alcohol. A $0.01 \mathrm{~mol} / \mathrm{l}$ citrate buffer was added for high-temperature antigen retrieval for $7 \mathrm{~min}$, followed by $3 \% \mathrm{H}_{2} \mathrm{O}_{2}$ for $10 \mathrm{~min}$. The sections were sealed with a sealant containing $10 \%$ goat serum at room temperature for $1 \mathrm{~h}$. The rabbit anti-rat polyclonal antibody (1:100) was added for overnight incubation of TLR4, MyD88 and NF-KB(p65) at $4{ }^{\circ} \mathrm{C}$. The antibody fixation of TLR4, MyD88 and NF$\kappa \mathrm{B}(\mathrm{p} 65)$ was detected using anti-rat/rabbit IHC kits (Proteintech, USA). After 2 min of restaining with haematoxylin, the tissue sections were dehydrated and sealed with neutral resins. The images were analysed using Image-J (National Institutes of Health, USA). Any three 200-fold fields were taken from each immunohistochemical film of each rat to measure the positive cumulative absorbance of the region.

\section{Detection by flow cytometry}

Cells taken from each group were mixed with $5 \mu \mathrm{l}$ of annexin V-fluorescein isothiocyanate (FITC) and $10 \mu \mathrm{l}$ of propidium iodide for staining. The cells were incubated in a dark place at room temperature for $15 \mathrm{~min}$. The samples were mixed with $400 \mu \mathrm{l}$ of buffer solution, filtered through a 300mesh nylon mesh and then placed on the EPICS XL instrument for flow cytometry analysis. The data were analysed using EXPO32 ADC.

\section{RT-qPCR}

After being treated, cells were collected from each group and then mixed with Trizol reagent homogenate. QIAzol lysis reagent was used to extract the total RNA. cDNA was synthesised by a cDNA reverse transcription kit. RT-qPCR was performed using the SYBR-Green PCR kit in accordance with the manufacturer's instructions. U6 was used an internal control for miR-145-5p and GAPDH for TLR4, MyD88 and NF-kB(p65). Changes in gene expression were calculated using the $2^{-\Delta \Delta c t}$ method. The sequences of the primers used are listed in Table I.

\section{Western blot assay}

When cells in each group were treated accordingly for $48 \mathrm{~h}$, the total protein was extracted and separated by sodium dodecyl sulphate-polyacrylamide gel electrophoresis on $10 \%$ polyacrylamide 
Table I. Primer sequences

\begin{tabular}{|lc|}
\hline Gene name & \multicolumn{1}{c|}{ Primer sequence } \\
\hline miR-145-5p & F: 5'-CCTTGTCCTCACGGTCCAGT-3' \\
\cline { 2 - 2 } & R: 5'-AACCATGACCTCAAGAACAGTATTT-3' \\
\hline U6 & F:5'- GCTTCGGCAGCACATATACTAAAAT -3' \\
\cline { 2 - 2 } R:5'- CGCTTCACGAATTTGCGTGTCAT -3'; & F:5'-AGACATCCAAAGGAATACTGCAA-3' \\
\cline { 2 - 2 } TLR4 & R:5'-GCCTTCATGTCTATAGGTGATGC-3' \\
\hline MyD88 & F:5'-CGCCTGTCTCTGTTCTTGAA-3' \\
\cline { 2 - 2 } & R:5'-TCCGCTTGTGTCTCCAGTT-3' \\
\hline NF-кB(p65) & F:5'-GAGAGCCCTTGCATCCTTTA-3' \\
\cline { 2 - 2 } & R:5'-CTTCCCTTTGGTCTTTCTGT-3' \\
\hline GAPDH & F:5'-GAGTCAACGGATTTGGTCGT-3' \\
\cline { 2 - 2 } & R:5'-TTGATTTTGGAGGGATCTCG-3' \\
\hline
\end{tabular}

gel. The protein was transferred onto the polyvinylidene fluoride membrane and sealed with $10 \%$ skimmed milk at room temperature for $1 \mathrm{~h}$. The cells were mixed with primary antibodies (TLR 4 $1: 500$, MyD88 $1: 1000$, NF- $\kappa B 1: 500$ and GAPDH 1 : 500), incubated overnight at $4^{\circ} \mathrm{C}$, mixed with goat anti-rabbit secondary antibody conjugated with horseradish peroxidase and then incubated for $1 \mathrm{~h}$. Protein bands were visualised with ECL exposure. Protein content was quantitatively analysed using the GelDoc. 2000 imaging system.

\section{Immunofluorescence staining of cells}

Cells were inoculated in 6-hole plates at $1 \times 10^{5 /}$ $2 \mathrm{ml}$ per hole by cell counting. The culture solution was discarded after culturing with whole culture medium for $24 \mathrm{~h}$, and then the medium was replaced with a culture medium containing only $0.5 \%$ FBS for $24 \mathrm{~h}$ pre-treatment to achieve cell synchronisation. Cells in all groups were treated for $48 \mathrm{~h}$ and then washed three times with PBS. The cells were fixed with $4 \%$ paraformaldehyde for $60 \mathrm{~min}$, washed three times with PBS, 5\% normal goat serum was added dropwise for sealing for $30 \mathrm{~min}$, rabbit $\mathrm{p}-\mathrm{NF}-\kappa \mathrm{B}(\mathrm{p} 65)$ monoclonal antibody (1 : 100) was added dropwise directly, followed by incubation at room temperature for $90 \mathrm{~min}$. After washing the cells three times with PBS, FITC-labelled goat anti-rabbit fluorescent secondary antibody $(1: 100)$ was added dropwise. The cells were incubated at room temperature for 60 min, washed three times with PBS, DAPI-labelled cell nucleus was added dropwise, followed by incubation in a dark place at room temperature for $10 \mathrm{~min}$. The mixture was washed three times with PBS. The number of nucleated cells for $\mathrm{p}-\mathrm{NF}$ $\kappa \mathrm{B}(\mathrm{P} 65)$ was observed directly under a fluorescence microscope. The DAPI-labelled cell nucleus was added. The cells were observed to be blue under a fluorescence microscope, and images were analysed using Image-J software.

\section{Detection of target relation by dual- luciferase experiment}

U87 cells were incubated in a 24-hole plate at a density of $2 \times 10^{4} /$ hole. Upon reaching $70 \%$ fusion, the cells were mixed with $100 \mathrm{ng}$ of TLR4 WT or TLR4 MUT luciferase vector and $50 \mathrm{nmol} / \mathrm{l}$ miR-145-5p or mimic. NC was added for co-transfection. After $48 \mathrm{~h}$, the cells were collected, and luciferase activity was evaluated using the dual-luciferase report analysis system. Each experiment was repeated at least three times.

\section{Statistical analysis}

Data were processed by SPSS18.0. Measurement data were expressed as mean $\pm \mathrm{SD}$. Samples were tested by one way ANOVA, and the results conformed to normal distribution. A significant difference was considered at $p<0.05$.

\section{Results}

\section{Impacts of Cro on cerebral infarction rate}

The white area is the infarction, and the red area is the normal brain tissue. No pale infarction was observed in the sham group, but a large pale infarction was found in the model group. The infarction volume in all Cro-treated groups decreased to different extents. The cerebral infarction rate in the model group was significantly higher than that in the NC group ( $p<0.001$, Figure 1$)$. The infarction rate in the Cro intervention groups was significantly lower than that in the model group $(p<0.05$, Figure 1). A significant dose-effect relation was observed among the Cro-treated groups ( $p<0.05$, Figure 1 ).

\section{Cell injury in tissues}

The cells in the cerebral cortex in the NC group had structural integrity, regular morphology and dense arrangement. However, the cells in the brain tissues in the model group had increased peripheral clearance, irregular nuclei, obvious in terstitial oedema, disordered arrangement, hyperchromatic nuclei and solid shrinkage, or eosinophilic degeneration. The number of injured cells has higher, and the cell injury in the Cro-treated groups was alleviated compared with the model group (Figure $2 \mathrm{~A}$ ). TUNEL detection results in Figure 2 B showed that the number of apoptotic cells was significantly greater in the model group than in the NC group $(p<0.001)$. Meanwhile, the number of apoptotic cells was significantly lower in the Cro-treated groups than in the model group ( $p<0.05$, respectively, Figure $2 \mathrm{~B}$ ). A significant 
NC

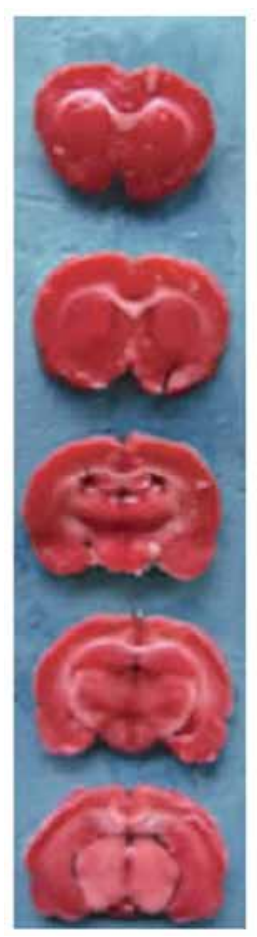

Model

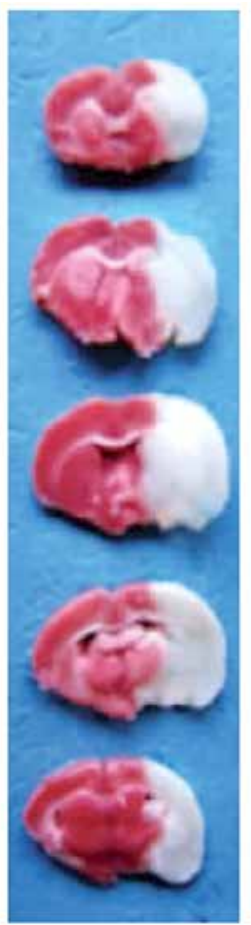

Cro-L

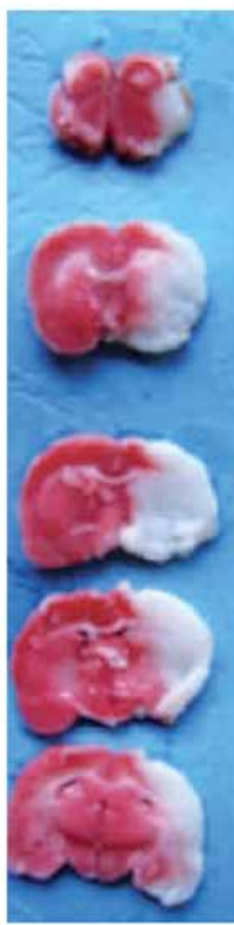

Cro-M

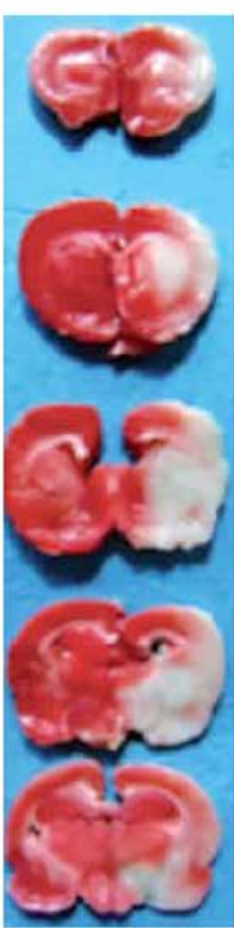

Cro-H

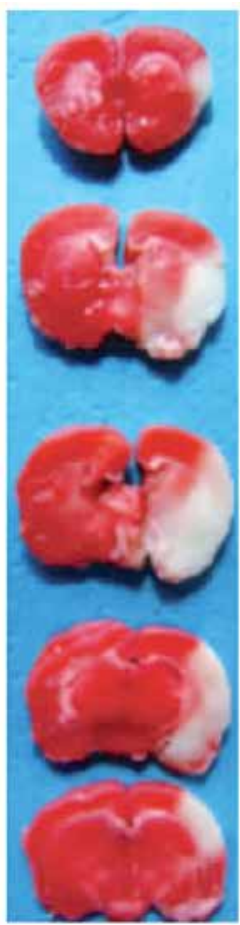

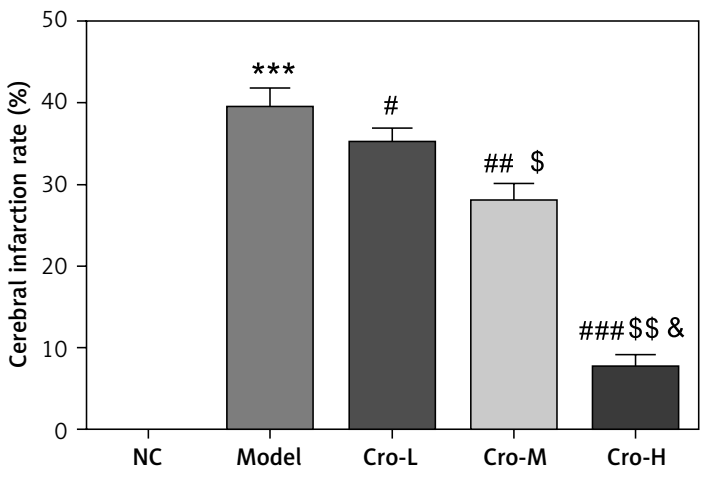

dose-effect relation was found among the groups $(p<0.05$, Figure $2 \mathrm{~B})$.

\section{Relative protein expression in different tissues by IHC assay}

The results of IHC assay showed that the protein expression levels of TLR4, MyD88 and NF$\kappa \mathrm{B}(\mathrm{p} 65)$ were significantly higher in the model group than in the NC group $(p<0.001$, Figures 3 $\mathrm{A}-\mathrm{C})$. Meanwhile, the protein expression levels of TLR4, MyD88 and NF-KB(p65) in the brain tissues significantly decreased in a dose-dependent manner in the Cro-treated groups compared with the model group ( $p<0.05$, Figures $3 \mathrm{~A}-\mathrm{C})$.

\section{Relative gene expression in different tissues by RT-qPCR assay}

The results of RT-qPCR assay revealed that the gene expression levels of TLR4, MyD88 and NF-
Figure 1. The cerebral infarction rate of different brain tissues by TTC staining

${ }^{* * *} P<0.001$, compared with NC group; ${ }^{*} p<0.05$, $\# p<0.01$, \#\# $p<0.001$, compared with model group; ${ }^{s} p<0.05$, ${ }^{s s} p<0.01$, compared with Cro-L group; ${ }_{p}<<0.05$, compared with Cro-M. $\kappa B(p 65)$ were significantly higher whereas that of miR-145-5p was significantly lower in the model group compared with the NC group $(p<0.001$, Figures $4 \mathrm{~A}-\mathrm{C}$ ). Meanwhile, the gene expression levels of TLR4, MyD88 and NF-KB(p65) significantly decreased whereas that of miR-145-5p significantly increased in a dose-dependent manner in the Cro-treated groups compared with the model group $(p<0.05$, Figures 4 A-C).

\section{Effect of Cro on cell apoptosis in vitro}

Flow cytometry demonstrated that the apoptosis rate was significantly up-regulated in the model group compared with the NC group $(p<0.001$, Figure 5). In vitro study showed that the cell apoptosis rate significantly improved in the Cro-treated groups in a dose-dependent manner $(p<0.05$, Figure 5). 
A

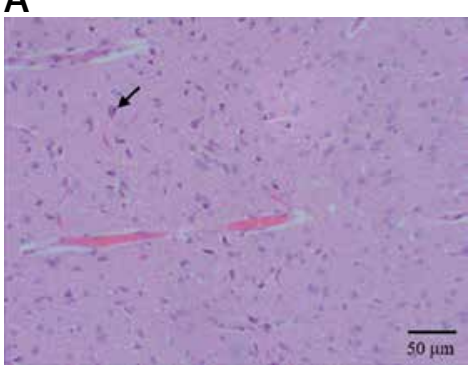

Model

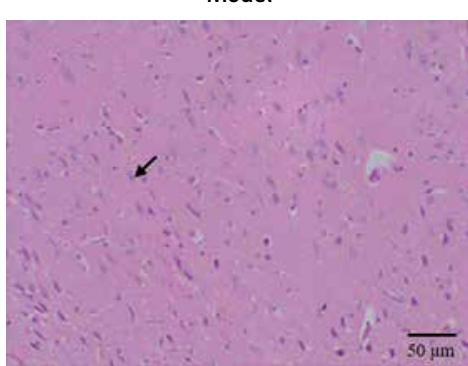

Cro-L

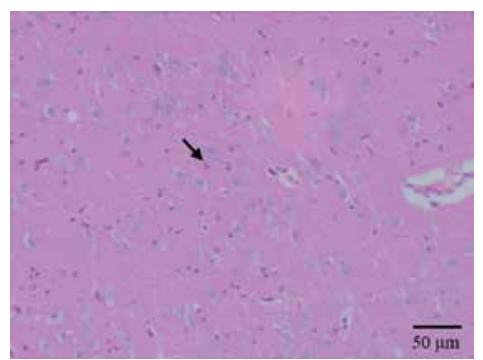

Cro-H
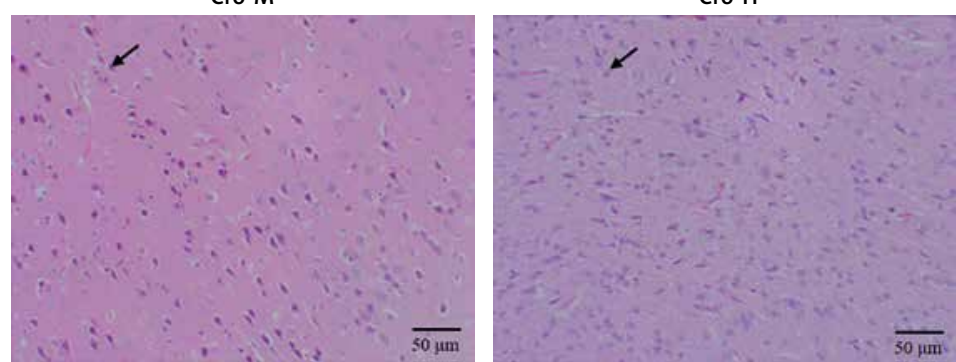

Model

Cro-L
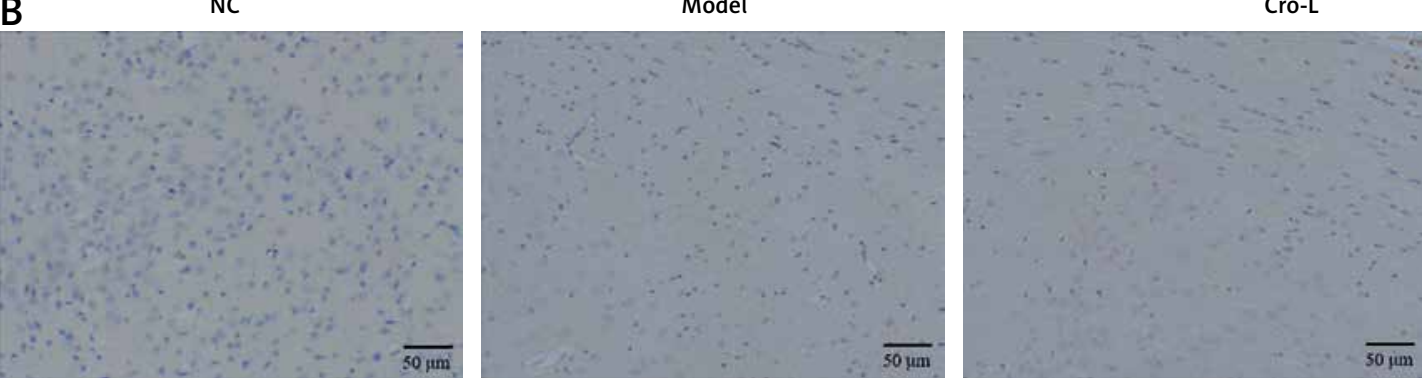

Cro-M

Cro-H
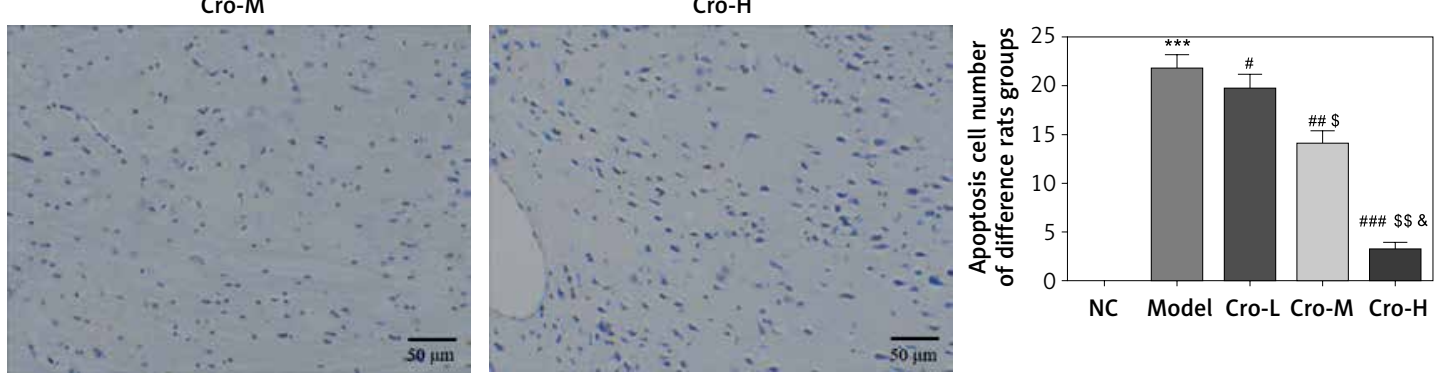

Figure 2. Cell injury in tissues. A - Pathological morphology of cerebral cortex in each group (200x). B - Apoptotic cells of different tissues in rats (200x)

${ }^{* * *} P<0.001$, compared with NC group; ${ }^{\#} p<0.05,{ }^{\# \#} p<0.01$, ${ }^{\# \# \#} p<0.001$, compared with model group; ${ }^{s} p<0.05,{ }^{S S} p<0.01$, compared with Cro-L group; ${ }^{*} p<0.05$, compared with Cro-M.

Effect of Cro on relative gene and protein expression levels by RT-qPCR and WB

MiR-145-5p gene expression was significantly lower and TLR4, MyD88 and NF-kB(p65) gene and protein expression levels were significantly higher in the model group compared with the NC group $(p<0.001$, Figures 6 A, B). MiR-145-5p gene expression significantly decreased and TLR4, MyD88 and NF-KB(p65) gene and protein expression levels significantly increased in the Cro-treated groups in a dose-dependent manner $(p<0.05$, respectively, Figures $6 \mathrm{~A}, \mathrm{~B}$ ).

\section{Effect of Cro on p-NF-kB(p65) nuclear volume}

Cellular immunofluorescence showed that the $\mathrm{p}-\mathrm{NF}-\mathrm{kB}(\mathrm{p} 65)$ nuclear volume significantly increased in the model group $(p<0.001$, Figure 7$)$. Cro supplementation significantly decreased the $\mathrm{p}-\mathrm{NF}-\mathrm{kB}(\mathrm{p} 65)$ nuclear volume in a dose-dependent manner in vitro $(p<0.05$, Figure 7$)$.

\section{Effect of miR-145-5p on Cro treatment}

The apoptosis rate was significantly higher in the model group than in the NC group $(p<0.001$, 
A

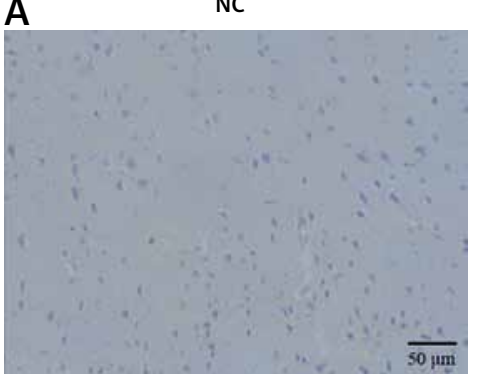

Cro-M

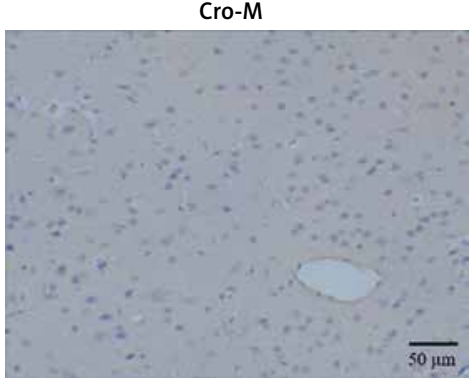

NC

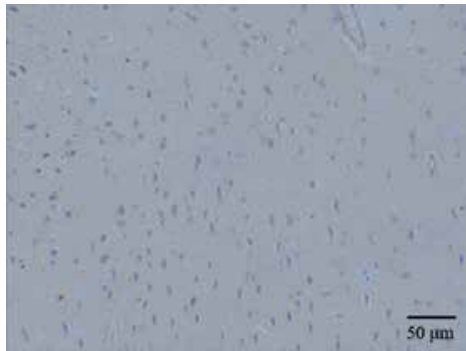

Cro-M

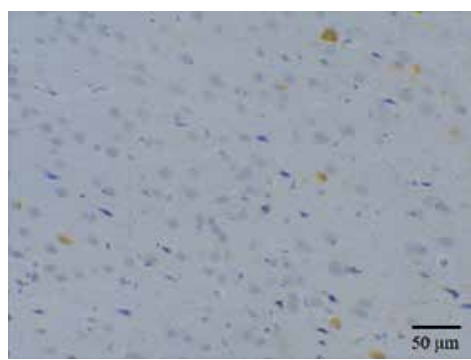

Model

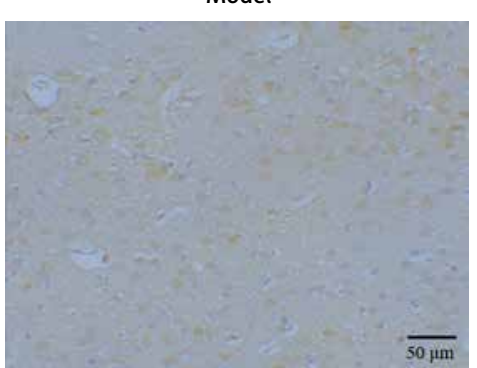

Cro-H

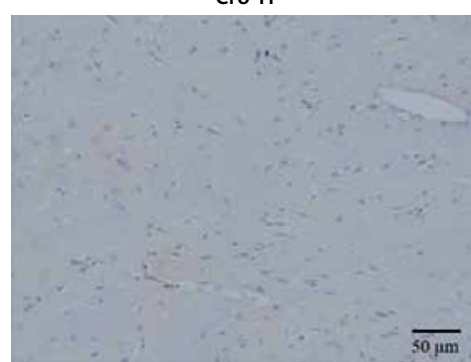

Model

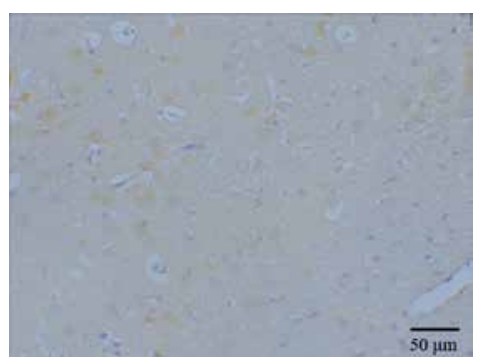

Cro-H

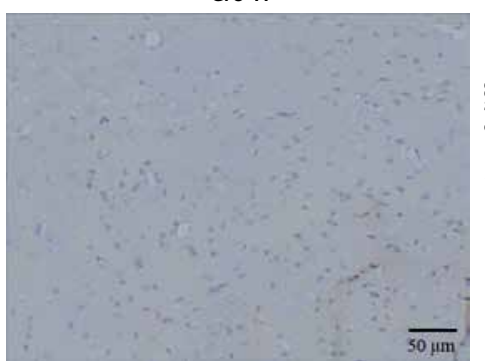

Cro-L
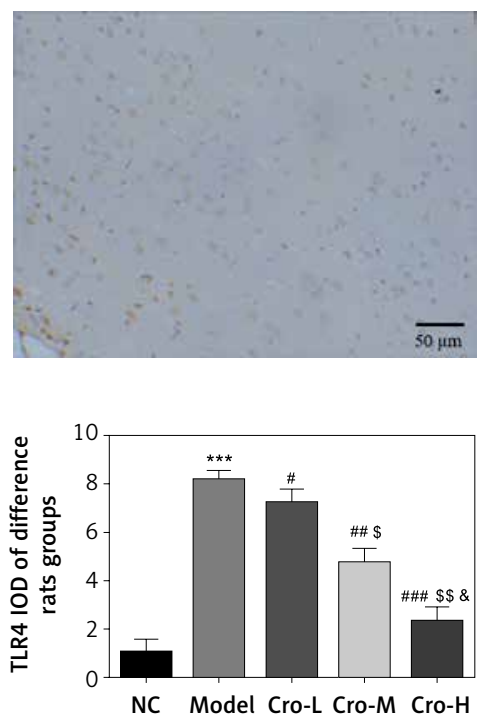

Cro-L
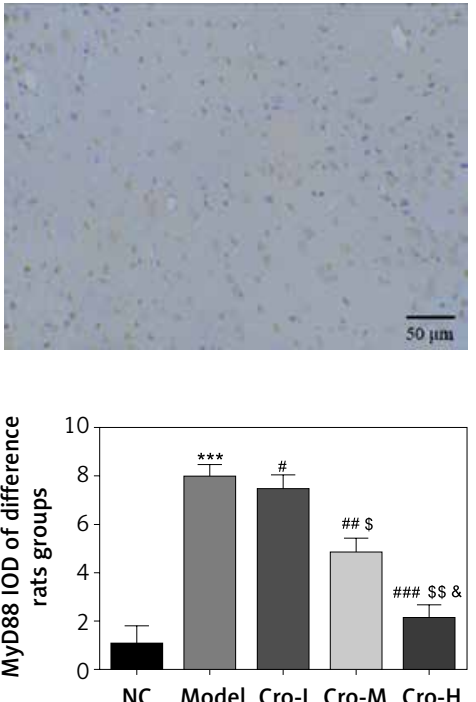

Figure 3. Relative protein expression in tissues by IHC assay (200x). A - TLR4 protein expression in brain tissues of different groups (200x). B - MyD88 protein expression in brain tissues of different groups (200x)

${ }^{* * *} p<0.001$, compared with NC group; ${ }^{*} p<0.05$, ${ }^{\# \#} p<0.01$, \#\# $p<0.001$, compared with model group; ${ }^{s} p<0.05,{ }^{s s} p<0.01$, compared with Cro-L group; ${ }^{*} p<0.05$, compared with Cro-M.

Figure 8). The apoptosis rate was significantly lower in the Cro-treated groups compared with the model group ( $p<0.001$, Figure 8 ). Meanwhile, the cell apoptosis rate of the $\mathrm{Cro}+$ miR-inhibitor group was significantly higher than those of the Cro-treated groups $(p<0.001$, Figure 8$)$.

\section{Effect of miR-145-5p on relative gene and protein expression}

MiR-145-5p gene expression was significantly lower and TLR4, MyD88 and NF-אB(p65) gene and protein expression levels were significantly higher in the model group compared with the NC group ( $p<0.001$, Figures 9 A, B). Cro supplement significantly increased miR-145-5p gene expression and significantly decreased TLR4, MyD88 and NF$\kappa \mathrm{B}(\mathrm{p} 65)$ gene and protein expression levels ( $p<$ 0.001 , Figures 9 A, B). MiR-145-5p gene expression was significantly lower and TLR4, MyD88 and NF- $\kappa \mathrm{B}(\mathrm{p} 65)$ gene and protein expression levels were significantly higher in the Cro + miR-inhibitor group compared with the Cor group $(p<0.001$, Figures 9 A, B). 


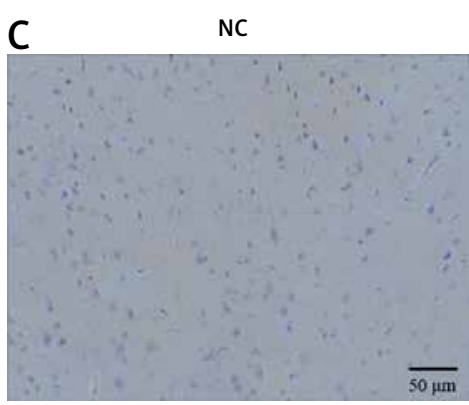

Cro-M

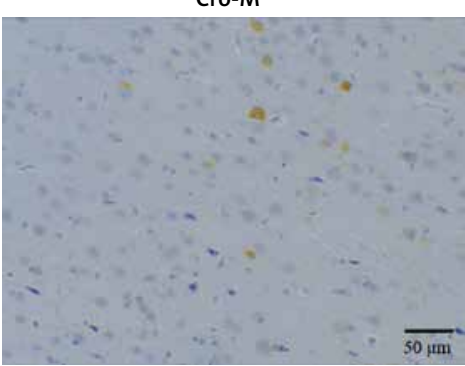

Model

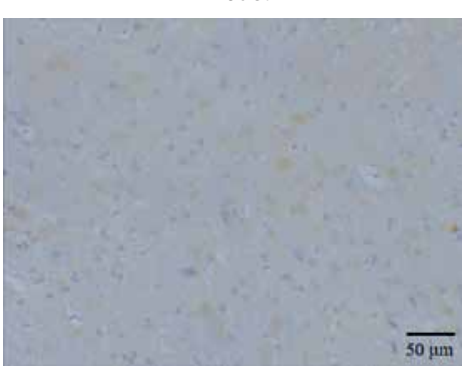

Cro-H

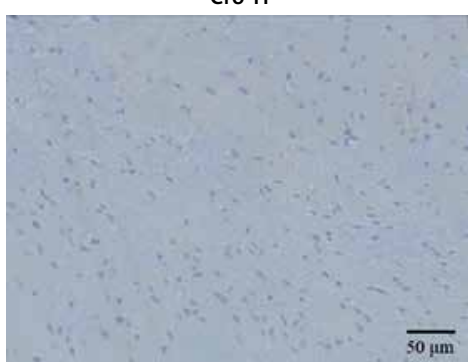

Cro-L
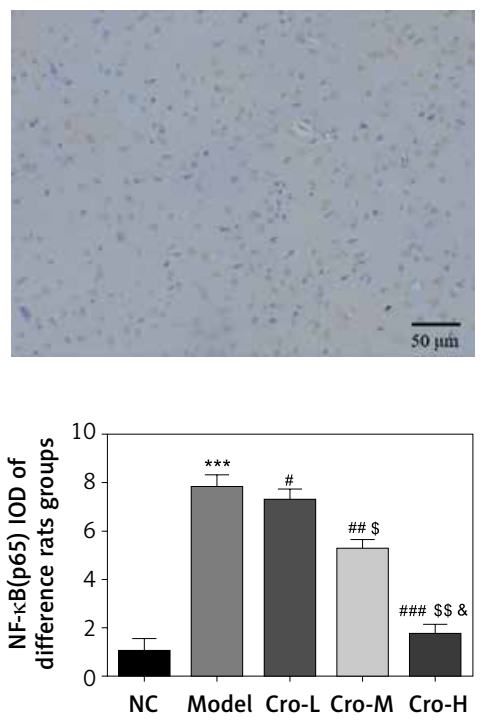

Figure 3. Cont. $\mathrm{C}-\mathrm{NF}-\kappa \mathrm{B}(\mathrm{p} 65)$ protein expression in brain tissues of different groups (200x)

${ }^{\star * *} p<0.001$, compared with NC group; ${ }^{\#} p<0.05,{ }^{\# \#} p<0.01$, \#\# $p<0.001$, compared with model group; ${ }^{s} p<0.05,{ }^{s 5} p<0.01$, compared with Cro-L group; ${ }^{\&} p<0.05$, compared with Cro-M.
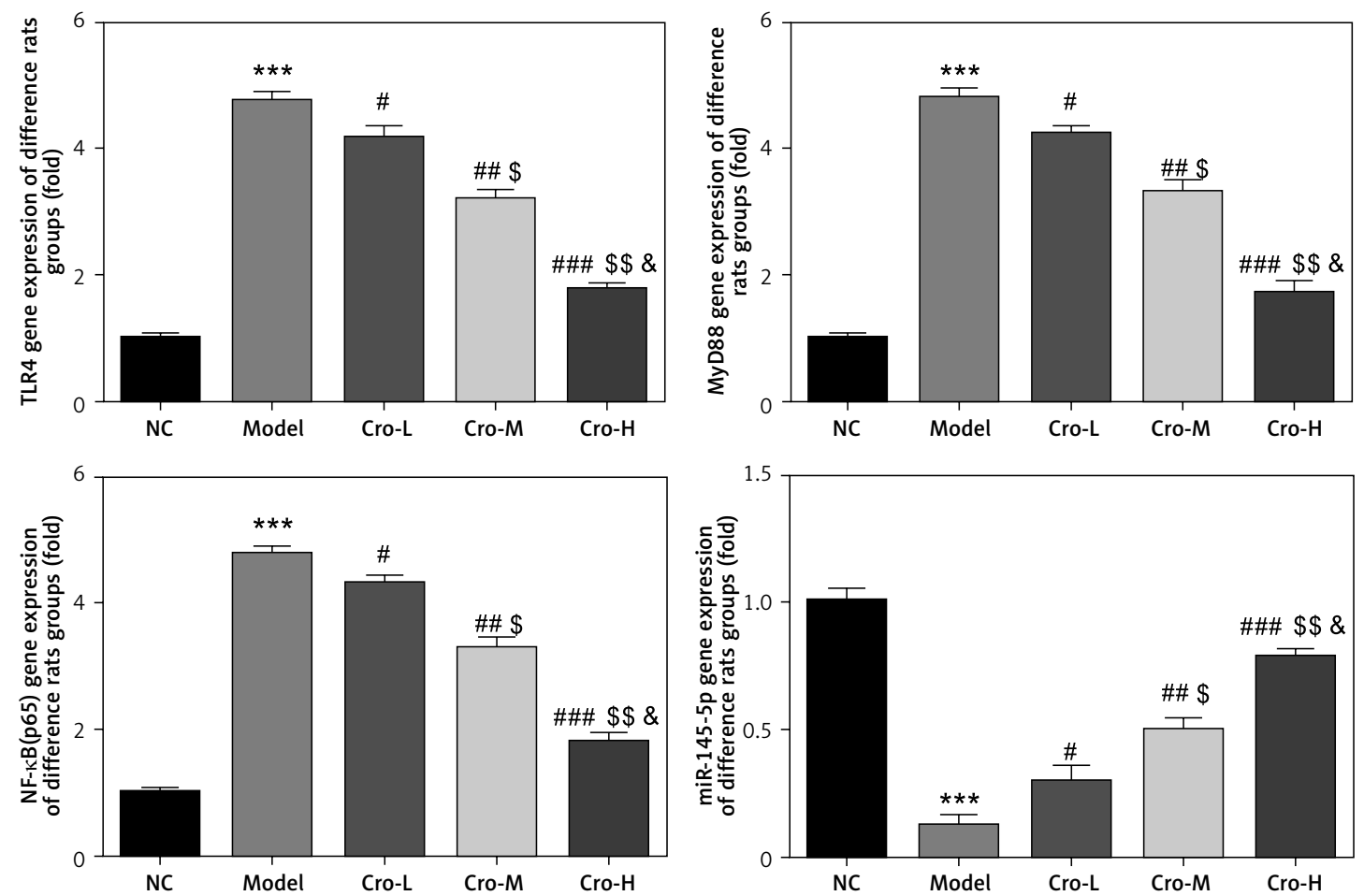

Figure 4. Relative gene expression in tissues by RT-qPCR

${ }^{\star * *} p<0.001$, compared with NC group; ${ }^{\#} p<0.05,{ }^{\# \#} p<0.01$, \#\#\# $p<0.001$, compared with model group; ${ }^{5} p<0.05,{ }^{\$ s} p<0.01$, compared with Cro-L group; ${ }^{\&} p<0.05$, compared with Cro-M. 

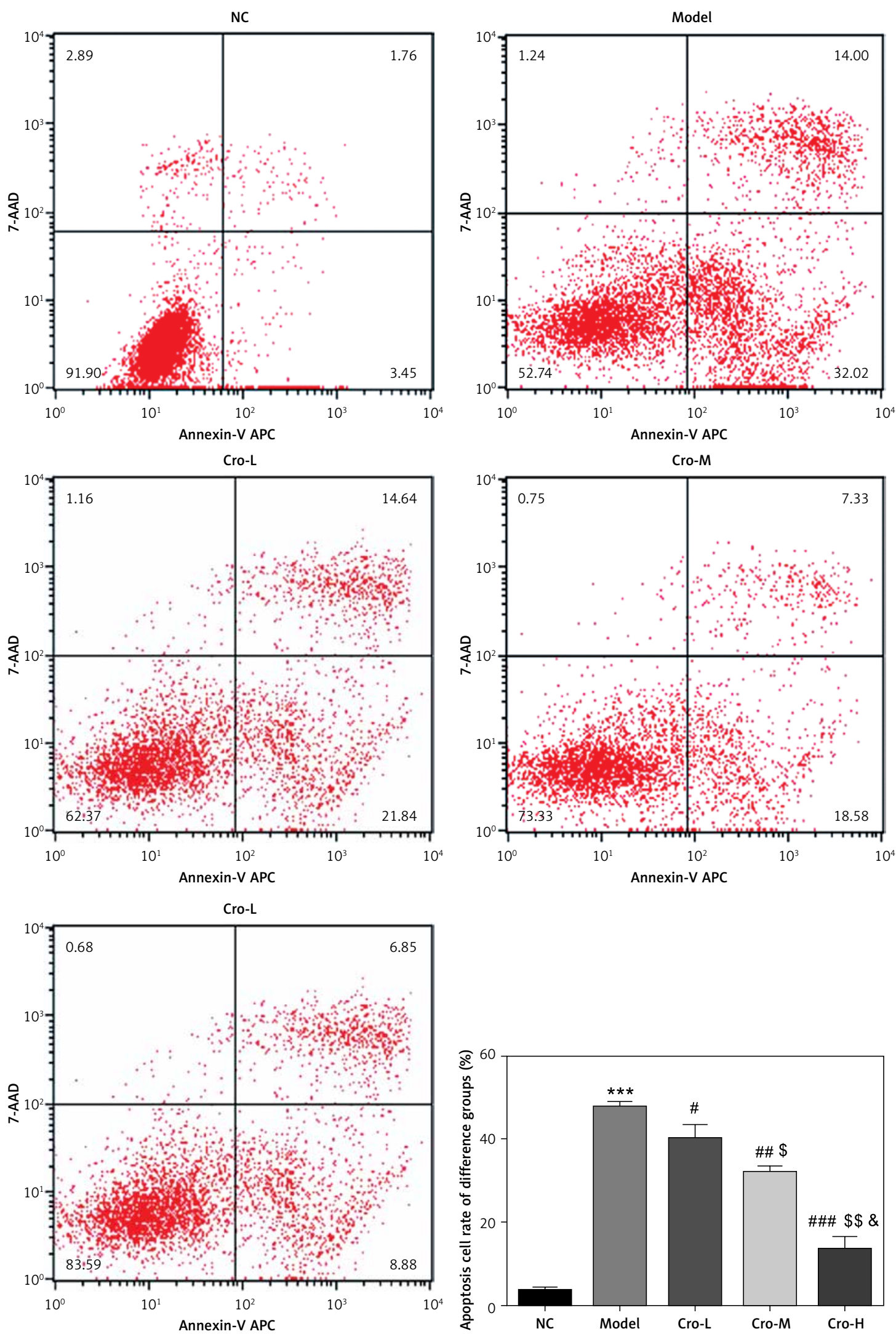

Figure 5. Cro improved cell apoptosis in vitro

${ }^{* * *} p<0.001$, compared with NC group; ${ }^{*} p<0.05,{ }^{* \#} p<0.01,{ }^{* \# \#} p<0.001$, compared with model group; ${ }^{s} p<0.05,{ }^{s 5} p<0.01$, compared with Cro-L group; ${ }^{\circledR} p<0.05$, compared with Cro-M. 

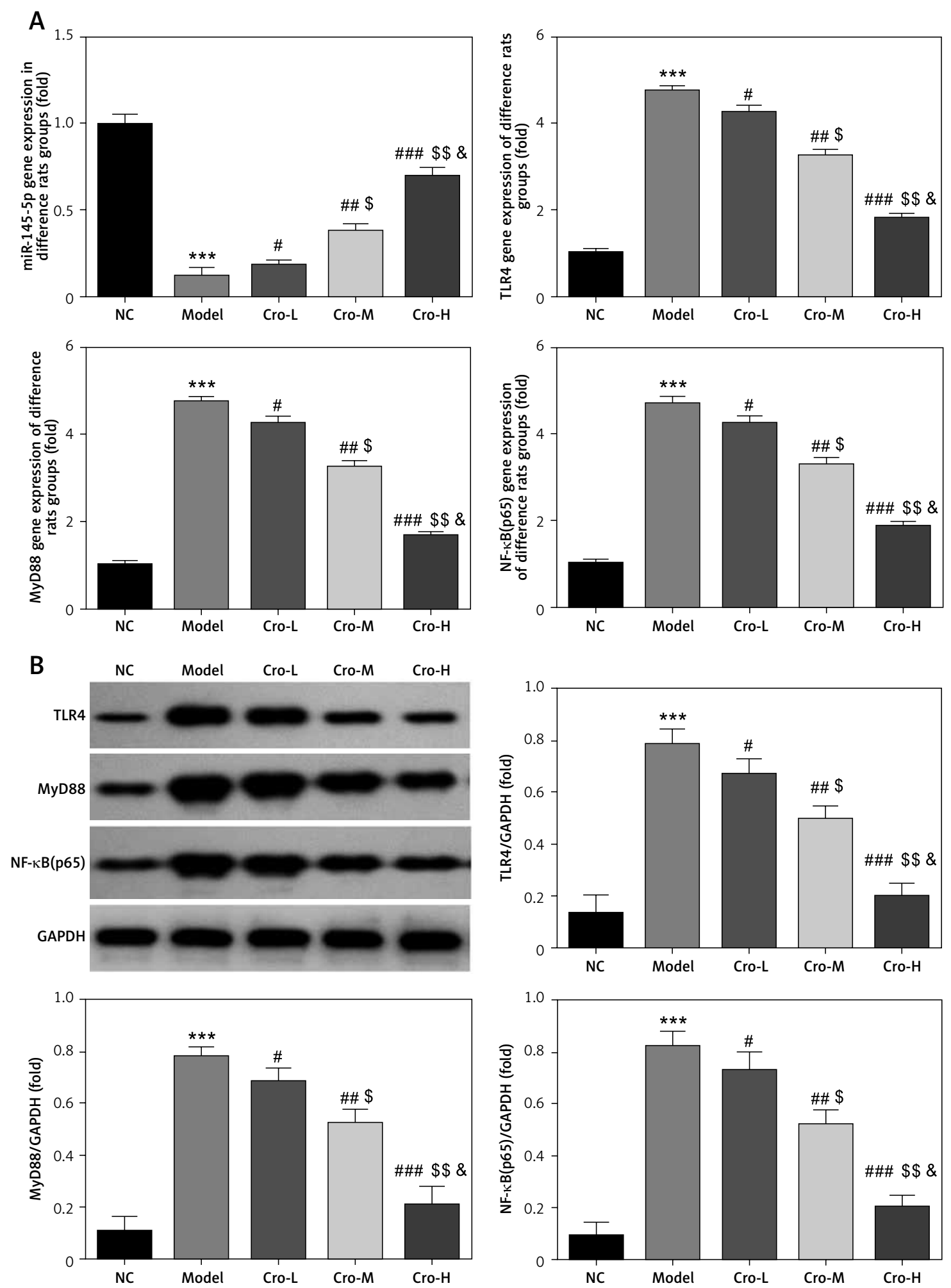

Figure 6. Relative gene and protein expression. A - Relative gene expression by RT-qPCR assay. B - Relative protein expressions by WB assay

${ }^{* * *} P<0.001$, compared with NC group; ${ }^{\#} p<0.05,{ }^{\# \#} p<0.01$, \#\#\# $p<0.001$, compared with model group; ${ }^{s} p<0.05,{ }^{s s} p<0.01$, compared with Cro-L group; ${ }^{\circ} p<0.05$, compared with Cro-M. 
DAPI
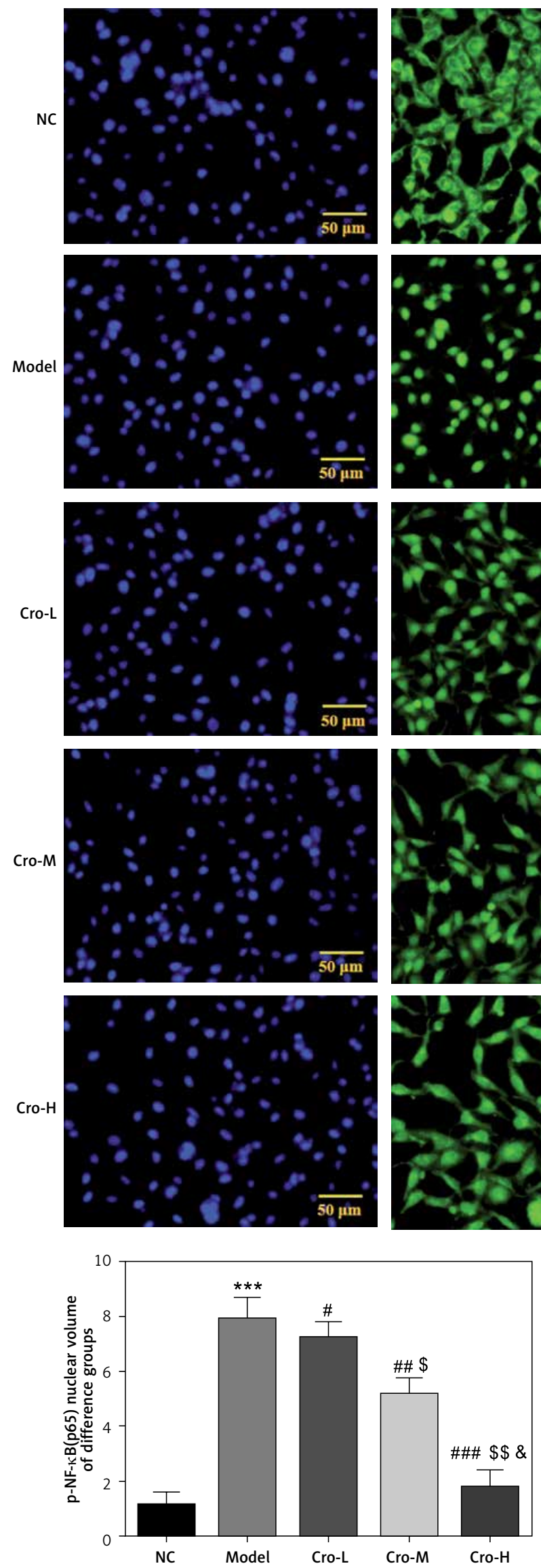

Merge
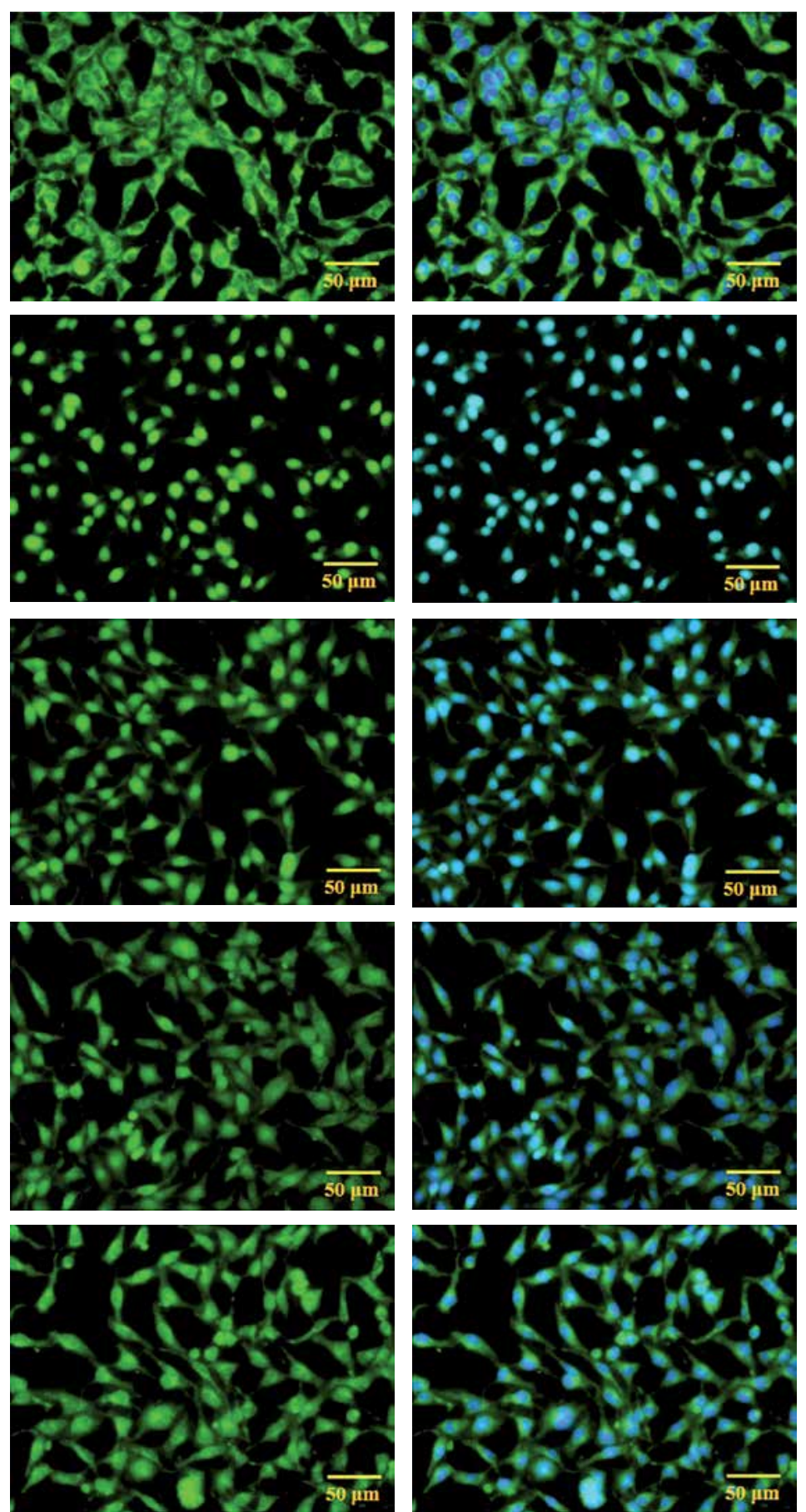

Figure 7. Cro affected p-NF- $\mathrm{B}(\mathrm{p65})$ nuclear volume $(200 x)$

${ }^{* * *} p<0.001$, compared with NC group; ${ }^{*} p<0.05$ $\# p<0.01$, \#\#p $<0.001$, compared with model group ${ }^{s} p<0.05$, ${ }^{s s} p<0.01$, compared with Cro-L group; $\& p<0.05$, compared with Cro-M. 

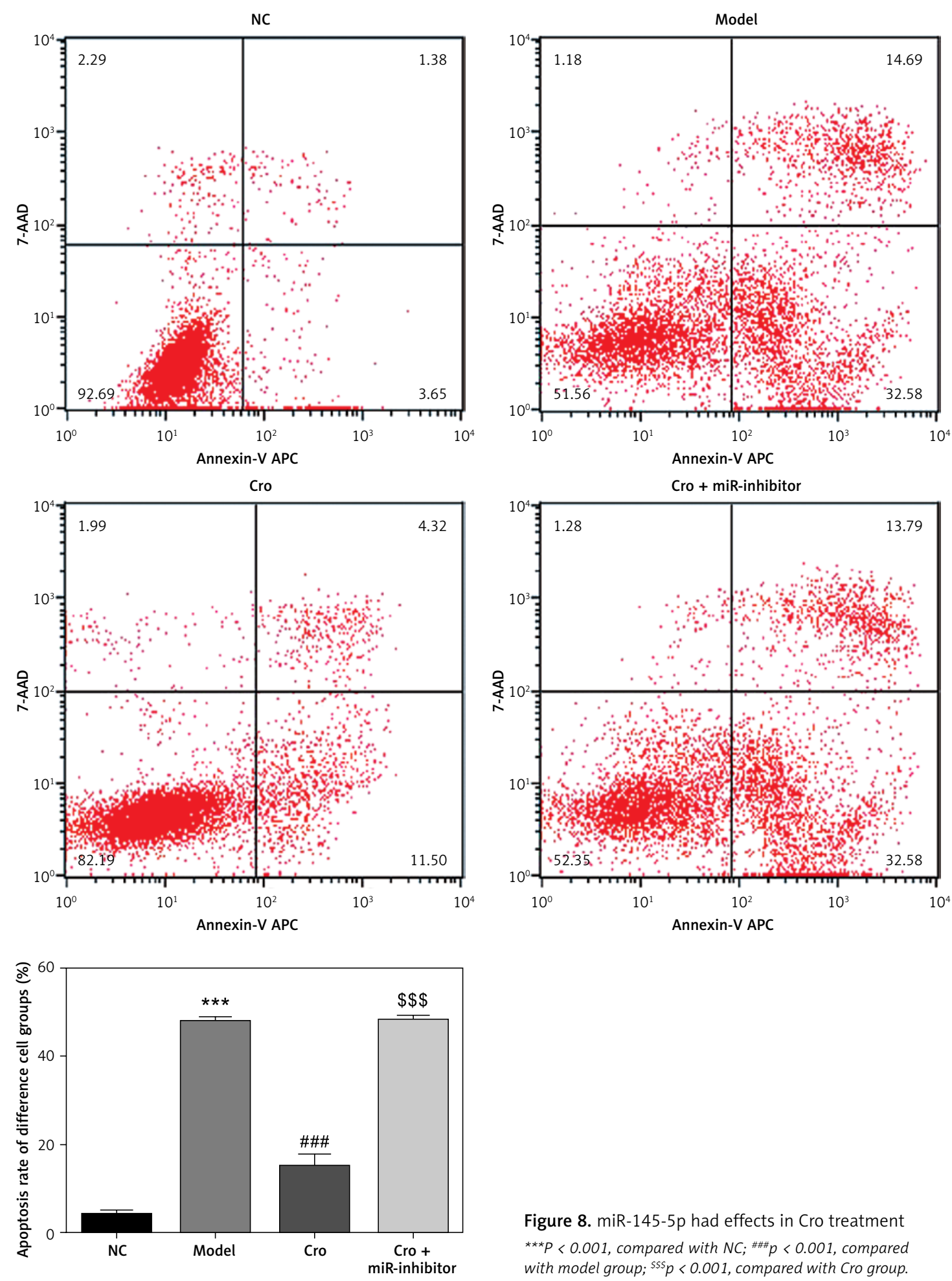

Figure 8. miR-145-5p had effects in Cro treatment

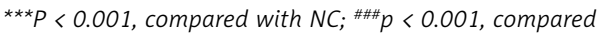
with model group; ${ }^{\$ \$ \$} p<0.001$, compared with Cro group. 

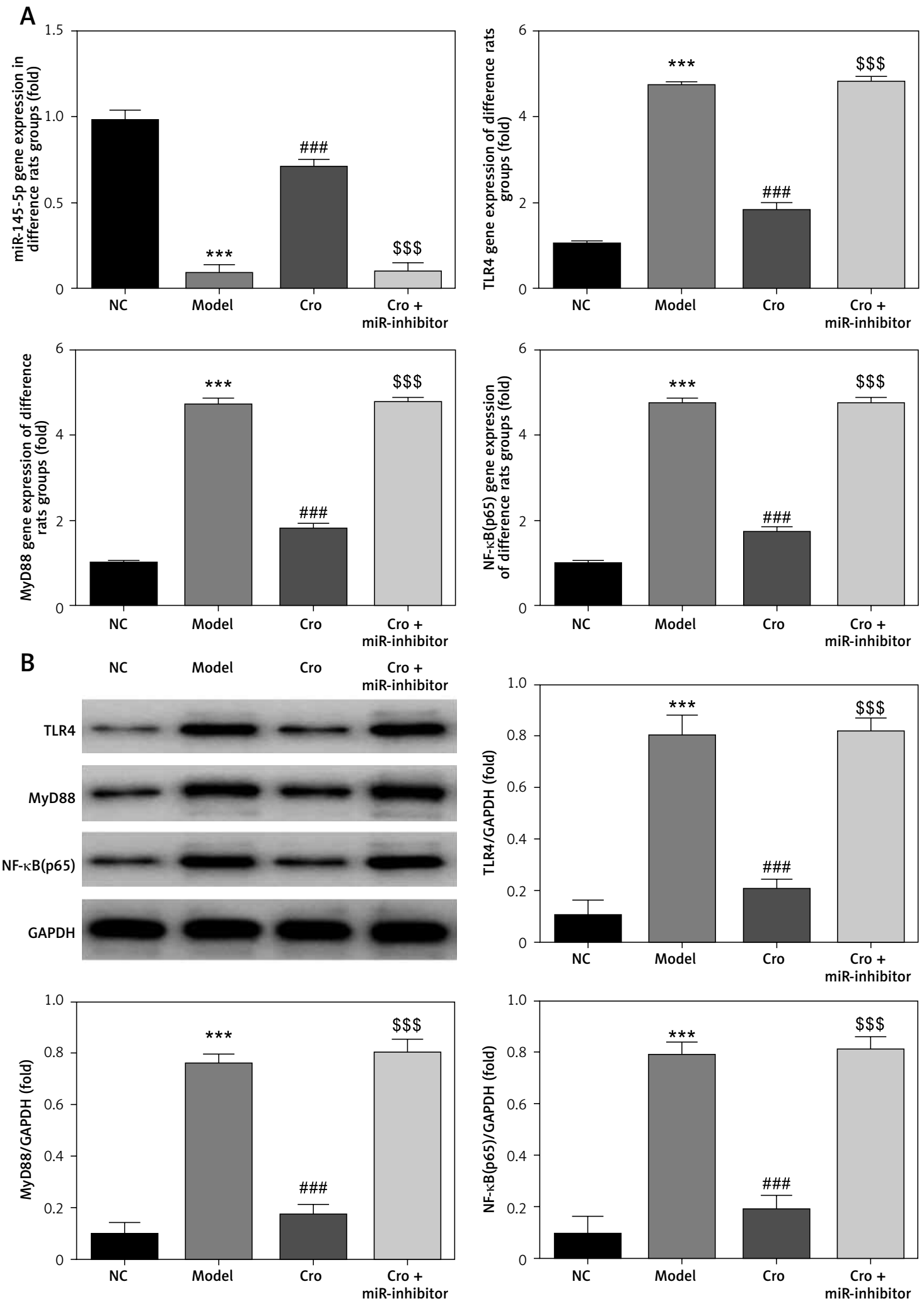

Figure 9. miR-145-5p had effects on relative gene and protein expression. A - Relative gene expression by RT-qPCR assay. B - Relative protein expressions by WB assay

${ }^{* * *} P<0.001$, compared with NC; ${ }^{\# \#} p<0.001$, compared with model group; ${ }^{s s s} p<0.001$, compared with Cro group. 


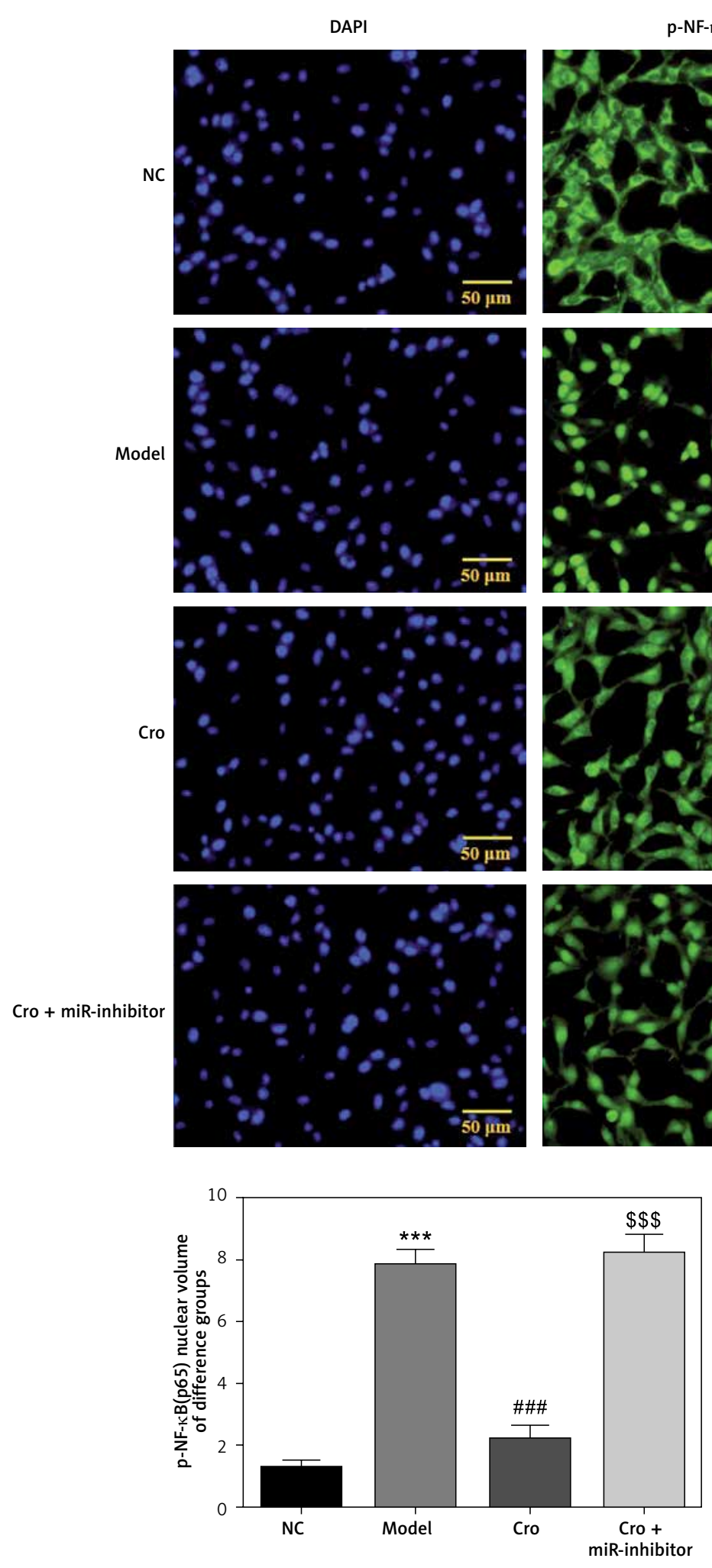

Effect of miR-145-5p on $\mathrm{p}-\mathrm{NF}-\mathrm{\kappa} B(\mathrm{p} 65)$ nuclear volume

The $\mathrm{p}-\mathrm{NF}-\kappa \mathrm{B}(\mathrm{p} 65)$ nuclear volume of the model group was significantly greater than that of the NC group ( $p<0.001$, Figure 10$)$. Meanwhile, this volume was significantly lower in the Cor-treated
Figure 10. miR-145-5p had effects on $p-N F-\kappa B(p 65)$ nuclear volume (200x)

${ }^{* * *} P<0.001$, compared with $N C ; \# \# p<0.001$, compared with model group; ${ }^{S S S} p<0.001$, compared with Cro group.

groups than in the model group $(p<0.001$, Figure 10). Furthermore, the p-NF- $\kappa B(p 65)$ nuclear volume was significantly greater in the $\mathrm{CrO}+$ miR-inhibitor group than in the Cor group ( $p<$ 0.001, Figure 10). 


\begin{tabular}{|l|l|l|l|l|l|l|l|l|}
\hline $\begin{array}{l}\text { Position 720-727 pf TLR4 3' UTR } \\
\text { hsa-miR-140-5p }\end{array}$ & $\begin{array}{l}\text { 5'... UCAAAUAUCCAUAUUAACCACUA... } \\
\text { 3' GAUGGUAUCCCAUUUUGGUGAC }\end{array}$ & IIIIII & $8 \mathrm{mer}$ & -0.38 & 98 & -0.38 & 2.581 & 0.41 \\
\hline
\end{tabular}

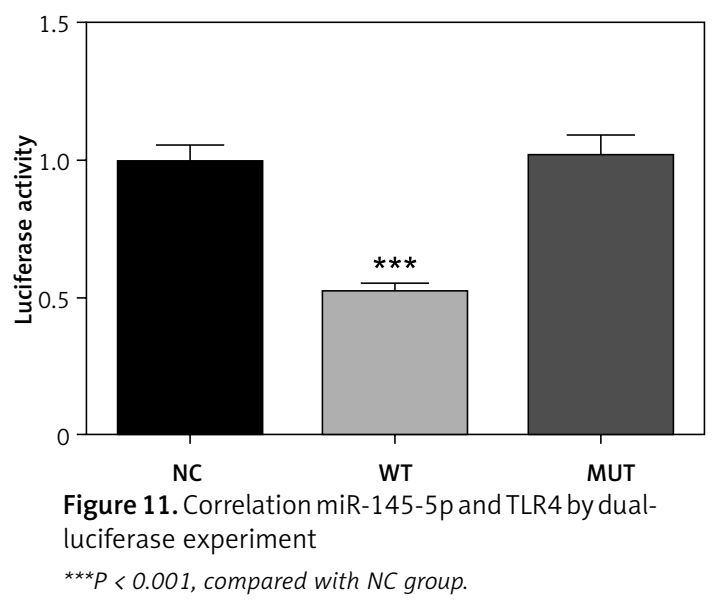

Correlation between miR-145-5p and TLR4 by dual-luciferase assay

The results of the dual-luciferase experiment revealed no significant difference between the NC and MUT groups. However, Luc activities were significantly lower in the WT group compared with the NC group ( $p<0.001$, Figure 11$)$. These results indicate that miR-145-5p targets TLR4 in U87 cells.

\section{Discussion}

In recent years, the effects of ncRNA on human diseases, such as tumours, cardiovascular disease and metabolic diseases, have attracted widespread attention. Many studies have shown that ncRNA is closely correlated with systemic or local inflammatory responses, cell proliferation, apoptosis and migration and directly participates in the occurrence and development of human diseases. miRNAs are ncRNAs involved in the pathogenesis of CIS. For instance, the expression of miR-19a in CIS is unusually low, whereas the high expression of miR-19 protects neurons from CIS by regulating glucose metabolism and inhibiting neuronal apoptosis [11]. Therefore, we speculate that CIS treatment may be accompanied by changes in miRNAs. Cro, the main chemical component of the stigma of $C$. sativus, can prevent atherosclerosis and reduce brain I/R injury. Ishizuka et al. [12] showed that Cro inhibits the ischaemic damage to the retina in mice by reducing oxidative stress and inhibiting apoptosis. Other studies proved that $\mathrm{Cro}$ can reduce the apoptosis of stem cells induced by experimental haemorrhagic shock [13] and inhibit the hyperglycaemic-induced apoptosis of human umbilical vein endothelial cells through the PI3K/ Akt/eNOS pathway [14]. However, the effects of Cro on CIS cell apoptosis have not been studied. The present study showed that CIS causes low ex- pression of miR-145-5p. This phenomenon may be correlated with brain cell damage caused by CIS. In addition, Cro intervention significantly inhibited the infarction rate in rat brain tissue and significantly increased the expression level of miR-145$5 p$. TargetScan prediction showed that the TLR4 3'UTR region has binding sites with miR-145-5p and verified the target relation by dual-luciferase experiment.

As innate immune receptors, TLRs play an important role in initiating secondary injury. In the central nervous system (CNS), TLR4 is expressed in the microglia and astrocyte glial progenitor cells, neurons and other cells [15]. Therefore, TLR4 may play a key role in regulating inflammation in the CNS. After cerebral infarction, large amounts of DAMPs bind to the TLR4 receptor to activate the downstream MyD88-dependent/non-MyD88-dependent signalling pathway, which activates the transcription factor NF- $\mathrm{KB}$ and the transcription of inflammatory or inflammation-related factors, and finally releases a large number of inflammatory cytokines and inflammatory chemokines, aggravating nerve injury [16]. The cerebral infarction volume can be significantly reduced by knocking out TLR4 or injecting TLR4 inhibitor, thus improving nerve function after cerebral infarction [17]. Phosphorylation of NF- $\kappa B(p 65)$ into the nucleus of $N F-\kappa B(p 65)$ downstream of TLR4/MyD88 is a key factor leading to cell apoptosis [18-20], and lowering the degree of NF- $\mathrm{KB}(\mathrm{p} 65)$ phosphorylation can effectively inhibit the cell apoptosis caused by inflammation [21-23]. In the present study, Cro intervention significantly decreased the amount of $\mathrm{p}-\mathrm{NF}-\mathrm{\kappa} \mathrm{B}(\mathrm{p} 65)$ entering the nucleus, which may be closely correlated with the improvement of CIS by Cro. However, the therapeutic effect of Cro disappeared after the transfection with miR-145-5p inhibitor. 
In conclusion, Cro can inhibit TLR4/MyD88/ NF- $\kappa B(p 65)$ by up-regulating miR-145-5p, thus reducing the amount of $p-N F-\kappa B(p 65)$ entering the nucleus and improving the cell apoptosis in the brain after $\mathrm{CIS}$.

However, there were some limitations in our study. miR-145-5p could target TLR4, while miR145-5p was one kind of miRNAs which target TLR4. There might be some other miRNAs in Cro improving CIS. This point will be investigated in our future study.

\section{Acknowledgments}

This study was supported by a talent Introduction Project of the Beijing Bureau of Foreign Expert (BJ2018001).

\section{Conflict of interest}

The authors declare no conflict of interest.

\section{References}

1. Wang W, Jiang B, Sun H, et al. Prevalence, incidence, and mortality of stroke in China: results from a nationwide population-based survey of 480687 adults. Circulation 2017; 135: 759-71.

2. Zhou M, Wang H, Zeng X, et al. Mortality, morbidity, and risk factors in China and its provinces, 1990-2017: a systematic analysis for the global burden of disease study 2017. Lancet 2019; 394: 1145-58.

3. Faralli A, Bigoni M, Mauro A, et al. Noninvasive strategies to promote functional recovery after stroke. Neural Plast 2013; 2013: 854597

4. Wang CJ, Lee MJ, Chang MC, et al. Inhibition of tumor promotion in benzo[a]pyrene-initiated CD-1 mouse skin by crocetin. Carcinogenesis 1995; 16: 187-91.

5. Hosseinzadeh H, Sadeghnia HR. Safranal, a constituent of Crocus sativus (saffron), attenuated cerebral ischemia induced oxidative damage in rat hippocampus. J Pharm Pharm Sci 2005; 8: 394-9.

6. Khalili M, Roghani M, Ekhlasi M. The effect of aqueous Crocus sativus $L$. extract on intracerebroventricular streptozotocin-induced cognitive deficits in rat: a behavioral analysis. Iranian J Pharm 2010; 8: 185-91.

7. Jou I, Lee JH, Park SY, et al. Gangliosides trigger inflammatory responses via TLR4 in brain glia. Am J Pathol 2016; 168: 1619-30.

8. Yao S, Tang B, Li G, et al. Mir-455 inhibits neuronal cell death by targeting TRAF3 in cerebral ischemic stroke. Neumpsychiatr Dis Treat 2016; 12: 3083-92.

9. Zhang L, Chopp M, Liu X, et al. Combination therapy with VELCADE and tissue plasminogen activator is neuroprotective in aged rats after stroke and targets microRNA-146a and the toll-like receptor signaling pathway. Arterioscler Theomb Vasc Biol 2012; 32: 1856-64.

10. Wu Z, Zhu Y, Cao X, et al. Mitochondrial toxic effects of $A \beta$ through mitofusins in the early pathogenesis of Alzheimer's disease. Mol Neurobiol 2014; 50: 986-96.

11. Ge XL, Wang JL, Liu X, et al. Inhibition of miR-19a protects neurons against ischemic stroke through modulating glucose metabolism and neurobal apoptosis. Cell Mol Biol Lett 2019; 24: 37.
12. Ishizuka F, Shimazawa M, Umigain N, et al. Crocetin, a carotenoid derivative, inhibits retinal ischemic damage in mice. Eur J Pharmacol 2013; 703: 1-10.

13. Ahrazem O, Diretto G, Gómez-Gomez L, et al. Evolutionarily distinct carotenoid cleavage dioxygenases are responsible for crocetin production in Buddleja davidi. J Exp Bot 2017; 68: 4663-77.

14. Yang R, Vernon K, Thomas A, et al. Crocetin reduces activation of hepatic apoptotic pathways and improves survival in experimental hemorrhagic shock. J Parenter Enteral Nutr 2011; 35: 107-13.

15. Pan N, Lu LY, Li M, et al. Xyloketal B alleviates cerebral infarction and neurologic deficits in a mouse stroke model by suppressing the ROS/TLR4/NF-kappa B inflammatory signaling pathway. Acta Pharmacol Sin 2017; 38: 1236-47.

16. Li LT, Zhan XJ, Cui LL, et al. Ursolic acid promotes the neuroprotection by activating Nrf2 pathway after cerebral isehemiain mice. Brain Res 2013; 1497: 32-9.

17. Chen NN, Wang JP, Liu HF, et al. The bone marrow mononuclear cells reduce the oxidative stress of cerebral infaretion through P13K/AKT/NRF2 signaling pathway. Eur Rev Med Pharmacol Sci 2017; 21: 5729-35.

18. Wei X, Sun C, Zhou RP, et al. Nerve growth factor promotes ASICla expression via the NF-KB pathway and enhances acid-induced chondrocyte apoptosis. Int Immunopharmacol 2020; 82: 106340.

19. Song $\mathrm{H}$, Xun $\mathrm{S}$, He H, et al. Compound porcine cerebroside and ganglioside injection (CPCGI) attenuates sevoflurane-induced nerve cell injury by regulating the phosphorylation of p38 MAP kinase (p38MAPK)/nuclear factor kappa B (NF-kB) pathway. Med Sci Monit 2020; 26: e919600.

20. Liu W, Cheng L, Li Q, et al. TRIP6 regulates the proliferation, migration, invasion and apoptosis of osteosarcoma cells by activating the NF-kappaB signaling pathway. Exp Ther Med 2020; 19: 2317-25.

21. Han X, Zhang LU, Liu Y, et al. Resveratrol protects H9c2 cells against hypoxia-induced apoptosis through miR30d-5p/SIRT1/NF-KB axis. J Biosci 2020; 45; 42.

22. Jiang $B$, Xue $M, X u D$, et al. Upregulation of microRNA-204 improves insulin resistance of polycystic ovarian syndrome via inhibition of HMGB1 and the inactivation of the TLR4/NF-KB pathway. Cell Cycle 2020; 19: 697-710.

23. Wang X, Li C, Wang Y, et al. UFL1 alleviates LPS-induced apoptosis by regulating the NF- $\mathrm{KB}$ signaling pathway in bovine ovarian granulosa cells. Biomolecules 2020; $10: 260$ 